\section{The NCE (Mean Field) Principle With Locality Dependent Cost Interactions}

\author{
Minyi Huang, Peter E. Caines, and Roland P. Malhamé
}

\begin{abstract}
We study large population stochastic dynamic games where each agent assigns individually determined coupling strengths (with possible spatial interpretation) to the states of other agents in its performance function. The mean field methodology [14] yields a set of decentralized controls which generates an $\varepsilon_{N}$-Nash equilibrium for the population of size $N$. A key feature of the mean field approximation (here with localized interactions) is that the resulting $k$ th individual agent's control law $u_{k}$ depends on that agent's state and the precomputable weighted average trajectory of the collection of all agents each applying a decentralized control law.
\end{abstract}

Index Terms-Decentralized control, large-population, linear-quadraticGaussian (LQG) control, Nash certainty equivalence (NCE) principle, Nash equilibrium, stochastic dynamic game.

\section{INTRODUCTION}

For noncooperative games with mean field coupling, the Nash Certainty Equivalence (NCE) methodology developed in our past work [13]-[16], [18] provides an effective analytical tool for obtaining decentralized strategies. The key idea of this methodology is to specify a certain consistency relationship between the individual strategies and the mass effect (i.e., the overall effect of the population on a given agent) within the population limit such that individual strategies are individually optimal responses to the mass effect and further replicate the same mass effect, and each player may ignore the fine details of any other individual player. This procedure leads to decentralized strategies for the individual players in a large but finite population. For this class of game problems, a closely related approach has recently been independently developed by Lasry and Lions [21], [22], while for models of many firm industry dynamics, Weintraub, Benkard, and Van Roy proposed the notion of oblivious equilibrium by use of mean field approximations [29], [30]. Long run average costs and horizon discounted costs were used in [23] and [31] for dynamic games with mean field coupling. For the analysis of mean field models in the setting of mathematical physics, see [9], [27]. To see the rich economic backgrounds of noncooperative games with many players, the reader is referred to [10], [11], [19], [20] and references therein.

Although mean field models in their usual uniform aggregation form have a broad scope of applications [4], [8], [13], [20], [22], they may be too limited to capture structural properties in certain problems. For instance, in a vaccination mean field model, each person assesses his

Manuscript received January 28, 2009; revised October 10, 2009; accepted August 09, 2010. Date of publication August 30, 2010; date of current version December 02, 2010. This technical note was presented in part at the IEEE CDC Conference, 2008. This work was supported in part by the Natural Sciences and Engineering Research Council of Canada (NSERC). Recommended by Associate Editor J.-F. Zhang.

M. Huang is with the School of Mathematics and Statistics, Carleton University, Ottawa, ON K1S 5B6, Canada (e-mail: mhuang@math.carleton.ca).

P. E. Caines is with the Department of Electrical and Computer Engineering and CIM, McGill University, Montreal, QC H3A 2A7, Canada (e-mail: peterc@cim.mcgill.ca).

R. P. Malhamé is with the Department of Electrical Engineering, École Polytechnique de Montréal, Montreal, QC H3C 3A7, Canada and also with the Group For Research In Decision Analysis (GERAD), Montréal, QC, H3T 2A7, Canada (e-mail: roland.malhame@polymtl.ca).

Color versions of one or more of the figures in this technical note are available online at http://ieeexplore.ieee.org.

Digital Object Identifier 10.1109/TAC.2010.2069410 or her infection risk and as a rough approximation may simply refer to the vaccination coverage of the overall population [4], [8], but in reality, the different sub-populations around the respective individuals may differently impact each person. It is obvious that an individual's close friends, colleagues (or classmates) have a much higher immediate influence than those more distant in a social and physical sense. A similar situation arises in economic models. In a crowded business area, a service unit (such as a retail store or a restaurant) and its nearby neighbors may strongly interact while the level of such interactions decreases with distance. In mathematical biology, distance dependent interaction models have been employed to study selfish herd behavior of animals [25].

It is worthwhile briefly reviewing the extent to which game theory has dealt with the issue of locality. Blume [7] considered strategic interactions on lattice models as motivated by retailing services. Schelling [26] presented a simple line topology to examine social segregation phenomena where each agent attempts to move to a more favorable location. Despite the fact that they involve very different contexts, a common feature of the above works is their investigation of the relationship between the microscopic local behavior of individual agents and the resulting macroscopic phenomena (also see, e.g., [6], [12], [24]).

Motivated by these problems, we present here a generalized mean field version of the Nash Certainty Equivalence theory of our previous work (see [13], [14], [16]-[18]) which now takes into account the possibility of the local nature of agent interactions. As in [14], our approach relies on identifying a certain consistency relationship between each individual and the mass effect but the latter may now be specific to individual agents. We will be particularly interested in addressing the validity issue of mean field approximations in relation to spatial decay rates of the interaction. The reader is referred to [28, pp. 95] and [3, pp. 60-63] for a similar validity problem in statistical mechanics. Namely, the mean field approximation is valid when the particles have sufficiently long interaction ranges.

The technical note is organized as follows. The individual dynamics and costs are introduced in Section II where the uniform aggregate cost coupling [13], [14] is reviewed; we also identify some novel features for locality based interactions by examining their tolerable decay rates so that the mean field approximation is valid. Section III presents the equilibrium analysis for the set of strategies calculated via the NCE equation system. Section IV gives a numerical example. Section V concludes the technical note.

\section{The Stochastic Dynamic Game Model}

In a population of $N$ agents, consider the dynamics for an individual agent

$$
d z_{i}(t)=\left[A_{i} z_{i}(t)+B_{i} u_{i}(t)\right] d t+D d W_{i}(t), \quad 1 \leq i \leq N, t \geq 0
$$

where $z_{i} \in \mathbb{R}^{n}, u_{i} \in \mathbb{R}^{m}$ is the control input, and $\left\{W_{i}, 1 \leq i \leq N\right\}$ denotes $N$ independent $n$ dimensional standard Wiener processes. The matrices $A_{i}, B_{i}$ and $D$ have compatible dimensions. The initial states $\left\{z_{i}(0), 1 \leq i \leq N\right\}$ are mutually independent and also independent of $\left\{W_{i}, 1 \leq i \leq N\right\}$. In addition, $E\left|z_{i}(0)\right|^{2}<\infty$. In (1), the pair of coefficients $\theta_{i}:=\left[A_{i}, B_{i}\right]$ will be called the dynamic parameter. The variability of $\theta_{i}$ is used to model a heterogenous population of players. Denote the state configuration $z=\left(z_{1}, \ldots, z_{N}\right)$, and the population average state $z^{(N)}=(1 / N) \sum_{i=1}^{N} z_{i}$.

\section{A. The NCE Principle With Mean Field Cost Coupling}

We begin by giving a brief summary of our previous modeling of cost coupling which dealt with scalar individual states and control inputs. 
Thus, in Section II-A we restrict our attention to the case $n=m=1$, and the dynamics are assumed to be of the form

$$
d z_{i}=a_{i} z_{i} d t+b u_{i} d t+\sigma d W_{i}, \quad 1 \leq i \leq N .
$$

The cost function is given as

$$
J_{i}^{0}=E \int_{0}^{\infty} e^{-\rho t}\left\{\left[z_{i}-\Phi^{0}\left(z^{(N)}\right)\right]^{2}+r u_{i}^{2}\right\}(t) d t
$$

where $\rho>0$ is a discount factor, $\Phi^{0}=\gamma\left(z^{(N)}+\eta\right), z^{(N)}=$ $(1 / N) \sum_{i=1}^{N} z_{i}, \gamma>0, r>0$ and $\eta \in \mathbb{R}$ is a constant. It should be noted that for this mean field coupling of the uniform aggregation form, $\Phi^{0}$ does not distinguish the ordering of the entries within $z=\left(z_{1}, \ldots, z_{N}\right)$.

Let $\Pi_{a}>0$ be the positive solution to the algebraic Riccati equation

$$
\rho \Pi=2 a \Pi-\left(b^{2} / r\right) \Pi^{2}+1 .
$$

For simplicity we assume zero initial mean for all agents, i.e., $E z_{i}(0)=0, i \geq 1$. Also, we assume that the sequence of parameters $\left\{a_{i}, i \geq 1\right\}$ is contained in a given compact set $\mathcal{A} \subset \mathbb{R}$ and has a limiting empirical distribution $F(a)$. The NCE consistency requirement leads to the equation system

$$
\begin{aligned}
\rho s_{a} & =\frac{d s_{a}}{d t}+a s_{a}-\left(b^{2} / r\right) \Pi_{a} s_{a}-z^{*} \\
\frac{d \bar{z}_{a}}{d t} & =\left[a-\left(b^{2} / r\right) \Pi_{a}\right] \bar{z}_{a}-\left(b^{2} / r\right) s_{a} \\
\bar{z} & =\int_{a \in \mathcal{A}} \bar{z}_{a} d F(a) \\
z^{*} & =\gamma(\bar{z}+\eta)
\end{aligned}
$$

where $\bar{z}_{a}(0)=0$ corresponds to the zero initial mean assumption. See [13], [14], [16] for details on the construction of this equation system in a linear-quadratic-Gaussian (LQG) context.

Under mild assumptions, the equation system (4)-(7) admits a unique bounded solution $\left(s_{a}(\cdot), \bar{z}_{a}(\cdot), \bar{z}(\cdot)\right)$. The function $s_{a}$ is uniquely determined by its boundedness condition and it is unnecessary to state the initial condition $s_{a}(0)$ separately. In fact, when the system consists of uniform agents (i.e., all $a_{i}$ 's take the same value $a$ ), $\bar{z}_{a}$ and $s_{a}$ may be solved explicitly ([16]). Denote the control law

$$
u_{i}^{0}=-\frac{b}{r}\left(\Pi_{a_{i}} z_{i}+s_{a_{i}}\right)
$$

which may be interpreted as the optimal tracking control law with respect to $z^{*}$ in place of $\Phi^{0}\left(z^{(N)}\right)$ in (2). It has been shown that the set of decentralized control laws $\left\{u_{i}^{0}, 1 \leq i \leq N\right\}$ results in an $\varepsilon$-Nash equilibrium, where $\varepsilon \rightarrow 0$ as $N \rightarrow \infty$. The formal definition of an $\varepsilon$-Nash equilibrium will be stated in Section III; also see [2].

\section{B. Illustrative Problem With Locality Dependent Coupling}

To motivate the general LQG game with locality dependent cost interactions, we consider the uplink power control problem for $N$ users in a single cell of a code-division multiple access (CDMA) wireless communication network. For simplicity of parametrization, we denote the cell by a circle of radius $R_{c}$ and the base station is located at its center. Let user $i$ be associated with a locality parameter $p_{i}=\left(r_{i}, \gamma_{i}\right)$. Here $r_{i}$ denotes the distance between the user and the base station, and $\gamma_{i}$ is a target signal-to-interference (SIR) ratio. Assume a system of $M$ service classes so that $\gamma_{i}$ can take one of $M$ different values, depending on the service class of user $i$. Suppose that the power level $x_{i}$ of user $i$ is adjusted by the rule

$$
d x_{i}=u_{i} d t+\sigma d w_{i}, \quad 1 \leq i \leq N
$$

where $u_{i}$ is the up/down adjustment of the power. The $N$ independent standard Wiener processes $\left\{w_{i}, 1 \leq i \leq N\right\}$ are used to model control uncertainty during signal amplification and transmission.

Let the power attenuation of user $i$ be denoted by $g\left(r_{i}\right)$ as a function of distance from the base station. We consider a large system. After matched filtering, the interference on user $i$ 's signal as received by the base station may be denoted by $(\beta / N) \sum_{j \neq i} g\left(r_{j}\right) x_{j}+\eta_{0}$, where $\eta_{0}$ is the background noise and where $\beta / N$ is due to the use of a spreading code of length proportional to $N$ [13]. Thus the SIR for user $i$ after matched filtering is

$$
\Gamma_{i}=\frac{g\left(r_{i}\right) x_{i}}{(\beta / N) \sum_{j \neq i} g\left(r_{j}\right) x_{j}+\eta_{0}} .
$$

The control objective is to have $\Gamma_{i} \approx \gamma_{i}$, or equivalently

$$
g\left(r_{i}\right) x_{i} \approx \gamma_{i}\left[(\beta / N) \sum_{j \neq i} g\left(r_{j}\right) x_{j}+\eta_{0}\right]
$$

Based on the requirement (11), we adopt the discounted quadratic cost function

$$
E \int_{0}^{\infty} e^{-\rho t}\left\{\left[x_{i}-\left(\sum_{j \neq i} c_{p_{i} p_{j}}^{(N)} x_{j}+\gamma_{i} \eta_{0}\left(g\left(r_{i}\right)\right)^{-1}\right)\right]^{2}+r u_{i}^{2}\right\} d t
$$

where $c_{p_{i} p_{j}}^{(N)}=\gamma_{i}(\beta / N)\left(g\left(r_{j}\right) / g\left(r_{i}\right)\right)$ and $r>0$. The penalty of $r u_{i}^{2}$ is used to prevent abrupt power change since in an uncertain environment power control should be cautiously performed. An important feature of the cost (12) is that each $x_{j}$ in the coupling term $\sum_{j \neq i} c_{p_{i} p_{j}}^{(N)} x_{j}$ has a weight $c_{p_{i} p_{j}}^{(N)}$ depending on both locality parameters $p_{i}$ and $p_{j}$.

\section{NCE Principle With Locality Dependent Cost Interactions}

Now we formulate the LQG game with locality dependent cost interactions and generalize the basic NCE equation system to this case. To this end, we assign the agents a "locality" (or "spatial") parameter. Note that this locality parameter may have different interpretations and is not necessarily required to be a physical location. For instance, it may be used to measure the relative locations of the players or their social distances in a social interaction context [1]. The locality parameter for agent $i$ is denoted by $p_{i}$.

Suppose that the system dynamics are given by (1). Let the cost for the $i$ th agent be given by

$$
J_{i}=E \int_{0}^{\infty} e^{-\rho t}\left\{\left[z_{i}-\Phi_{i}\right]^{T} Q\left[z_{i}-\Phi_{i}\right]+u_{i}^{T} R u_{i}\right\} d t
$$

where $\rho>0, \Phi_{i}=\gamma\left(\sum_{j=1}^{N} \omega_{p_{i} p_{j}}^{(N)} z_{j}+\eta\right), \gamma>0, \eta \in \mathbb{R}^{n}, Q \geq 0$ and $R>0$. We make the assumption:

(A1) The weight allocation satisfies:
i) $\omega_{p_{i} p_{j}}^{(N)} \geq 0, \forall i, j$;
ii) $\sum_{j=1}^{N} \omega_{p_{i} p_{j}}^{(N)}=1, \forall i$;
iii) $\lim _{N \rightarrow \infty} \sup _{1 \leq i \leq N} \sum_{j=1}^{N}\left[\omega_{p_{i} p_{j}}^{(N)}\right]^{2}=0$.

For each fixed $i$,(A1)-ii) stipulates that the total weight of unit is allocated to the $N$ agents. Compared to (12), the use of this unit total weight 
condition will simplify our asymptotic analysis. For notational simplicity, the summation in (A1)-ii) includes the index $i$ itself. Whether or not this self-weight is included has no impact on our asymptotic analysis when $N \rightarrow \infty$. The interpretation of (A1)-iii) is that the weights are not allowed to highly concentrate on a small number of agents. This restriction is important for obtaining good mean field approximations.

Example A: Let $p_{i}, 1 \leq i \leq N$, denote $N$ locations, uniformly spaced on the interval $[0,1]$, where $p_{i}=(i-1) /(N-1)$. Take $\omega_{p_{i} p_{i}}^{(N)}=0$ for each $i$ and

$$
\omega_{p_{i} p_{j}}^{(N)}=|j-i|^{-\lambda} / c_{i}, \quad 1 \leq i \neq j \leq N
$$

where $\lambda \in[0,1]$ and $c_{i}=\sum_{j=1, j \neq i}^{N}|j-i|^{-\lambda}$ is the normalizing factor.

For all $\lambda \in[0,1]$ in Example A, (A1) can be verified by elementary calculations. The mean field model of the uniform aggregation form corresponds to taking $\lambda=0$ so that equal weights are assigned to all other locations.

Example B: Consider the two dimensional grid $G d$ generated by partitioning the square $[0,1] \times[0,1]$ to obtain $N=(M+1)^{2}$ points of the form $p_{i j}=(i / M, j / M)$, where $0 \leq i, j \leq M$. Define the distance between two points in the $\operatorname{grid} G d:\left\|p_{i j}-p_{k, l}\right\|=\max \{|i-k|,|j-l|\}$. Let the weight allocation be given by the rule: $\omega_{p_{i j}, p_{k l}}^{(N)}=c_{i j} \| p_{i j}-$ $p_{k l} \|^{-\lambda}$ for two distinct points, and $\omega_{p_{i j}, p_{i j}}^{(N)}=0$, where $c_{i j}$ is the normalizing factor so that all weights add up to one.

We can show that for all $\lambda \in[0,2]$, condition (A1) is satisfied by Example B.

We consider a representative agent with dynamic parameter $\theta=$ $[A, B]$, and locality parameter denoted by $\alpha$ belonging to a compact set $\mathcal{C} \subset \mathbb{R}^{d_{0}}$. For instance, in Examples $\mathrm{A}$ and $\mathrm{B}$, we may take $\mathcal{C}=$ $[0,1]$ and $\mathcal{C}=[0,1] \times[0,1]$, respectively. Denote the state process of this agent by $z_{\theta, \alpha}(t)$ to explicitly indicate the associated dynamic and locality parameters, and denote its mean trajectory by $\bar{z}_{\theta, \alpha}(t)=$ $E z_{\theta, \alpha}(t)$, where $t \geq 0$.

For $\theta_{i}=\left[A_{i}, B_{i}\right]$, suppose that the sequence $\left\{\theta_{i}, i \geq 1\right\}$ is contained in a compact set $\Theta$ and has the limiting empirical distribution $F(\theta)$. Concerning notation, whenever $\theta$ (or $\theta_{i}$ ) is associated with the distribution function $F$, we make the convention that $\theta$ is naturally identified as a vector in $\mathbb{R}^{d}$ with $d=n(n+m)$, and accordingly $\Theta$ is interpreted as a subset of $\mathbb{R}^{d}$. For an agent associated with the locality parameter $\alpha$ (to be called an $\alpha$-agent), let its limiting weight allocation to other agents at different locations $\alpha^{\prime} \in \mathcal{C}$ be described by a probability distribution $F_{\alpha}\left(\alpha^{\prime}\right)$ (with all probability mass concentrated on $\mathcal{C}$ ) when $N$ goes to infinity. At any given location, we assume independence between the weight allocation to neighbors and the distribution of their individual dynamic parameters, as shown by the following approximation for an agent with a locality parameter $p_{i} \in \mathcal{C}$ within a large population:

$$
\left.\sum_{j=1}^{N} \omega_{p_{i} p_{j}}^{(N)} 1_{\left(p_{j} \in S, \theta_{j} \in H\right)} \approx \int_{\alpha^{\prime} \in S} \int_{\theta \in H} d F(\theta) d F_{\alpha}\left(\alpha^{\prime}\right)\right|_{\alpha=p_{i}}
$$

for $S \subset \mathcal{C}$ and $H \subset \Theta$. The product measure $d F(\cdot) d F_{\alpha^{\prime}}(\cdot)$ in (15) indicates that in the limiting model the assignment of weights to different locations $\alpha^{\prime}$ is independent of the distribution of the dynamic parameter $\theta$. We will formalize related conditions in assumption (A4).

Denote the algebraic Riccati equation

$$
\rho \Pi=A^{T} \Pi+\Pi A-\Pi B R^{-1} B^{T} \Pi+Q .
$$

Suppose that (16) has a positive definite solution $\Pi_{\theta}$, where $\theta=[A, B]$. Define

$$
A_{1}=A-B R^{-1} B^{T} \Pi_{\theta}, \quad A_{2}=A-B R^{-1} B^{T} \Pi_{\theta}-\rho I .
$$

(A2) $F_{\alpha}(w): \mathcal{C} \times \mathbb{R}^{d_{0}} \rightarrow[0,1]$ satisfies: i) $F_{\alpha}(\cdot)$ is a probability distribution function for each fixed $\alpha$ and $\int_{\alpha^{\prime} \in \mathcal{C}} d F_{\alpha}\left(\alpha^{\prime}\right)=1$; ii) $\int_{\alpha^{\prime} \in S} d F_{\alpha}\left(\alpha^{\prime}\right)$ is a measurable function of $\alpha$ for each Borel subset $S$ of $\mathcal{C}$; iii) $F_{\alpha^{\prime \prime}}(\cdot)$ converges to $F_{\alpha}(\cdot)$ weakly when $\alpha^{\prime \prime} \rightarrow \alpha$, where $\alpha$ and $\alpha^{\prime \prime}$ are in $\mathcal{C}$.

(A3) For each $\theta=[A, B]$ from the compact set $\Theta$, the pair $[A, B]$ is controllable and the pair $\left[Q^{1 / 2}, A\right]$ is observable. In addition, $A_{1}$ defined by (17) is asymptotically stable for all $\theta=[A, B] \in \Theta$.

Under (A3), (16) has a unique positive definite solution $\Pi_{\theta}$, and $A_{2}$ is always asymptotically stable.

For the given $\alpha$-agent, it is subject to the aggregate effect of other agents described by

$$
\bar{r}(\alpha, t)=\int_{\theta \in \Theta, \alpha^{\prime} \in \mathcal{C}} \bar{z}_{\theta, \alpha^{\prime}}(t) d F(\theta) d F_{\alpha}\left(\alpha^{\prime}\right)
$$

which, subject to appropriate conditions, is an approximation of $\sum_{j=1}^{N} \omega_{p_{i} p_{j}}^{(N)} z_{j}$ for sufficiently large $N$.

For simplicity, we assume that each agent has zero initial mean. Now, based on the individual and weighted mass interaction consistency relationship, we can derive the following new Nash Certainty Equivalence (Mean Field) (NCE) equation system

$$
\begin{aligned}
\rho s_{\theta, \alpha} & =\frac{d s_{\theta, \alpha}}{d t}+A^{T} s_{\theta, \alpha}-\Pi_{\theta} B R^{-1} B^{T} s_{\theta, \alpha}-R_{\alpha} \\
\frac{d \bar{z}_{\theta, \alpha}}{d t} & =\left(A-B R^{-1} B^{T} \Pi_{\theta}\right) \bar{z}_{\theta, \alpha}-B R^{-1} B^{T} s_{\theta, \alpha} \\
\bar{r}(\alpha, t) & =\int_{\theta^{\prime} \in \Theta_{, \alpha^{\prime} \in \mathcal{C}}} \bar{z}_{\theta^{\prime}, \alpha^{\prime}}(t) d F\left(\theta^{\prime}\right) d F_{\alpha}\left(\alpha^{\prime}\right), \\
R_{\alpha}(t) & =\gamma[\bar{r}(\alpha, t)+\eta] .
\end{aligned}
$$

The initial condition for (19) is $\bar{z}_{\theta, \alpha}(0)=0$ due to the zero initial mean assumption. We observe that when the distribution function $F_{\alpha}(\cdot)$ is independent of $\alpha,(18)-(21)$ reduces to the NCE equation system with standard mean field coupling without differentiation between neighbors. This holds since in this case $\bar{r}(\alpha, t)$ and hence $R_{\alpha}(t)$ are both independent of $\alpha$ (see Acknowledgments).

In the construction of individual strategies, each agent needs to know the distribution function $F(\cdot)$ and the family of distribution functions $\left\{F_{\alpha}(\cdot), \alpha \in \mathcal{C}\right\}$, but it is not required to know specific information on a particular neighbor, such as its dynamic parameter or its weight allocation in the space $\mathcal{C}$.

The system (18)-(21) is constructed such that an $\alpha$-agent carries out optimal tracking of the local mass effect $R_{\alpha}$ which, in turn, depends on locality related coupling as expressed in (20). Similar to the procedure in [14], (19) is obtained by taking expectation of the closed-loop equation of the $\alpha$-agent. A consistent solution to the NCE equation system consists of a triple $\left(s_{\theta, \alpha}(t), \bar{z}_{\theta, \alpha}(t), \bar{r}(\alpha, t)\right)$, where $\theta \in \Theta, \alpha \in \mathcal{C}$ and $t \in \mathbb{R}^{+}$. Each entry in the pair $\left(s_{\theta, \alpha}(t), \bar{z}_{\theta, \alpha}(t)\right)$ will be viewed as a function from $\Theta \times \mathcal{C} \times \mathbb{R}^{+}$to $\mathbb{R}^{n}$, and $\bar{r}(\alpha, t)$ will be treated as a function from $\mathcal{C} \times \mathbb{R}^{+}$to $\mathbb{R}^{n}$.

Define $C_{b}\left[\mathcal{C} \times \mathbb{R}^{+}\right]=\{f(\alpha, t) \mid f \in C[\mathcal{C} \times$ $\left.\left.\mathbb{R}^{+}\right],|f|:=\sup _{\alpha, t}|f(\alpha, t)|<\infty\right\}$, where $f$ is $n$ dimensional.

Let $A_{1}$ and $A_{2}$ be defined by (17). For each fixed $\alpha$, if $\bar{r}(\alpha, t)$ is given as a bounded continuous function of $t$, we solve a unique bounded solution $s_{\theta, \alpha}(t)$ from (18) to obtain

$$
\begin{aligned}
s_{\theta, \alpha}(t) & =-e^{-A_{2}^{T} t} \int_{t}^{\infty} e^{A_{2}^{T} \tau} R_{\alpha}(\tau) d \tau \\
& =-e^{-A_{2}^{T} t} \int_{t}^{\infty} e^{A_{2}^{T} \tau} \gamma[\bar{r}(\alpha, \tau)+\eta] d \tau .
\end{aligned}
$$


Next

$$
\begin{aligned}
\bar{z}_{\theta, \alpha^{\prime}}(t)= & \int_{0}^{t} e^{A_{1}(t-s)} B R^{-1} B^{T} e^{-A_{2}^{T} s} \\
& \times \int_{s}^{\infty} e^{A_{2}^{T} \tau} \gamma\left[\bar{r}\left(\alpha^{\prime}, \tau\right)+\eta\right] d \tau d s \\
= & :\left[\Gamma_{\theta} \bar{r}\left(\alpha^{\prime}, \cdot\right)\right](t)
\end{aligned}
$$

where $\Gamma_{\theta}$ is viewed as an operator acting on bounded continuous functions of $t \in[0, \infty)$. Finally

$$
(\Gamma \bar{r})(\alpha, t):=\int_{\theta \in \Theta} \int_{\alpha^{\prime} \in \mathcal{C}}\left[\Gamma_{\theta} \bar{r}\left(\alpha^{\prime}, \cdot\right)\right](t) d F_{\alpha}\left(\alpha^{\prime}\right) d F(\theta) .
$$

For any $f \in C_{b}\left[\mathcal{C} \times \mathbb{R}^{+}\right], \Gamma_{\theta} f(\alpha, \cdot)$ and $\Gamma f$ are defined in a similar manner.

In order to solve the NCE equation system (18)-(21), a key step is to find a fixed point $\bar{r}$ in a suitable function space for the operator $\Gamma$, i.e.

$$
(\Gamma \bar{r})(\alpha, t)=\bar{r}(\alpha, t) .
$$

Lemma 1 below is proven in Appendix.

Lemma 1: Under (A2)-(A3), $\Gamma$ is a mapping from $C_{b}\left[\mathcal{C} \times \mathbb{R}^{+}\right]$to $C_{b}\left[\mathcal{C} \times \mathbb{R}^{+}\right]$.

Let $C_{b}[0, \infty)$ denote the set of $\mathbb{R}^{n}$-valued bounded and continuous functions on $[0, \infty)$. Define the linear operator $\bar{\Gamma}$ on $C_{b}[0, \infty)$

$$
\begin{aligned}
(\bar{\Gamma} \zeta)(t)=\gamma \int_{\theta \in \Theta} \int_{0}^{t} e^{A_{1}(t-s)} B & R^{-1} B^{T} \\
& \times \int_{s}^{\infty} e^{A_{2}^{T}(\tau-s)} \zeta(\tau) d \tau d s d F(\theta)
\end{aligned}
$$

which can be shown to be a linear bounded operator from $C_{b}[0, \infty)$ to $C_{b}[0, \infty)$ and where we recall that $\theta=[A, B]$. Note that $\bar{\Gamma}$ is obtained from $\Gamma_{\theta}$ by dropping $\eta$ in (22) and next averaging with respect to $\theta$. Denote the norm of $\bar{\Gamma}$ by $\|\bar{\Gamma}\|$. We have $\|\bar{\Gamma}\|<1$ for $\gamma$ suitably small. Below we use a scalar model to illustrate the estimation of $\|\bar{\Gamma}\|$.

Example $C$ : Assume $B \equiv 1, Q=R=\rho=1$, and $A$ has uniform distribution on $[0, a]$, where $a>0$. It is straightforward to show that (A3) is satisfied. Furthermore, by (24) we obtain

$$
\begin{aligned}
\sup _{t \geq 0}|(\bar{\Gamma} \zeta)(t)| \leq & \sup _{t \geq 0}|\zeta(t)| \gamma\left(B^{2} / R\right) \int_{\theta}\left(1 /\left|A_{1} A_{2}\right|\right) d F(\theta) \\
= & (2 / \sqrt{3})(\gamma / a) \\
& \quad \times \sup _{t \geq 0}|\zeta(t)| \int_{0}^{a} \frac{1}{(A-1 / 2)^{2}+3 / 4} d A \\
= & (2 / \sqrt{3})(\gamma / a) \\
& \times\{\arctan (1 / \sqrt{3}) \\
& \quad+\arctan [(2 / \sqrt{3})(a-1 / 2)]\} \\
& \times \sup _{t \geq 0}|\zeta(t)| \\
\leq & 2.42(\gamma / a) \sup _{t \geq 0}|\zeta(t)| .
\end{aligned}
$$

When $a$ in Example $\mathrm{C}$ increases, individual agents are likely to have more unstable open loop dynamics. The interesting fact here is that a large $a$ actually helps to reduce the magnitude of $\|\bar{\Gamma}\|$. The reason is that the more unstable open loop will force the agents to apply a large control gain, resulting in a large stability margin for the closed-loop dynamics.

Theorem 2: If (A2)-(A3) hold and $\|\bar{\Gamma}\|<1$, there exists a unique bounded solution $\left(s_{\theta, \alpha}(\cdot), \bar{z}_{\theta, \alpha}(\cdot), r_{\alpha}(\cdot)\right)$ to the NCE equation system (18)-(21).

Proof: By Lemma 1, we see that $\Gamma$ is an operator from $C_{b}\left[\mathcal{C} \times \mathbb{R}^{+}\right]$ to itself, and $C_{b}\left[\mathcal{C} \times \mathbb{R}^{+}\right]$is a Banach space under the norm $|f|=$ $\sup _{\alpha, t}|f(\alpha, t)|$.

We take $f_{1}, f_{2} \in C_{b}\left[\mathcal{C} \times \mathbb{R}^{+}\right]$. By straightforward calculation, we obtain the estimate

$$
\left|\Gamma f_{1}-\Gamma f_{2}\right| \leq\|\bar{\Gamma}\| \cdot\left|f_{1}-f_{2}\right| .
$$

It follows that $\Gamma$ is a contraction. So there is a unique solution $\bar{r} \in$ $C_{b}\left[\mathcal{C} \times \mathbb{R}^{+}\right]$satisfying (23). Once the above $\bar{r}$ is obtained, we may obtain the other two entries in $\left(s_{\theta, \alpha}(t), \bar{z}_{\theta, \alpha}(t), \bar{r}(\alpha, t)\right)$. Uniqueness of the solution can be easily verified by using uniqueness of the fixed point to (23).

\section{MEAN FIELD SOLUtion ANALYSIS}

For the equilibrium analysis, we need the assumption:

(A4) The $p_{i}$-parametrized joint empirical distribution function

$$
\left.G_{p_{i}}^{(N)}(\alpha, \theta)=\sum_{j=1}^{N} \omega_{p_{i} p_{j}}^{(N)} 1_{\left(p_{j}<\alpha, \theta j\right.}<\theta\right)
$$

satisfies the condition: for each bounded and continuous function $\phi(\alpha, \theta)$

$$
\lim _{N \rightarrow \infty}\left|\int \phi(\alpha, \theta) d G_{p_{i}}^{(N)}(\alpha, \theta)-\int \phi(\alpha, \theta) d F_{p_{i}}(\alpha) d F(\theta)\right|=0
$$

uniformly with respect to $p_{i}$. In the indicator function of (25), each inequality holds componentwise if it involves vectors.

The above condition implies that $G_{p_{i}}^{(N)}(\alpha, \theta)$ weakly converges to the distribution function $F_{p_{i}}(\alpha) F(\theta)$; the reader is referred to [5] for related notion on weak convergence.

Suppose that we have $N$ agents with identical dynamic parameters and the weight allocation is given by Example A. If $\lambda=1$, we can show that (A4) is satisfied and in this case $F_{p_{i}}(x)=1$ if $x>p_{i}$, $F_{p_{i}}(x)=0$ if $x \leq p_{i}$, where $F_{p_{i}}$ is specified in (A2) and (A4). For $\lambda \in[0,1),(\mathbf{A 4})$ holds with $F_{p_{i}}$ being continuous.

\section{A. Properties of the NCE Based Control Laws}

Within the population of $N$ agents, for any $1 \leq k \leq N$, the $k$ th agent's admissible control set $\mathcal{U}_{k}$ consists of all feedback controls $u_{k}$ adapted to the $\sigma$-algebra $\sigma\left(z_{i}(\tau), \tau \leq t, 1 \leq i \leq N\right)$ (i.e., $u_{k}(t)$ is a function of $\left.\left(t, z_{1}(t), \ldots, z_{N}(t)\right)\right)$ such that a unique strong solution to the closed-loop system exists on $[0, \infty)$. Note that the strategies in $\mathcal{U}_{k}$ may use full state information. Denote $u_{-i}=\left(u_{1}, \ldots, u_{i-1}, u_{i+1}, \ldots, u_{N}\right)$. To indicate the dependence of $J_{i}$ on $u_{i}$ and $u_{-i}$, we write it as $J_{i}\left(u_{i}, u_{-i}\right)$.

Definition 3: A set of controls $u_{k} \in \mathcal{U}_{k}, 1 \leq k \leq N$, for $N$ players is called an $\varepsilon$-Nash equilibrium with respect to the costs $J_{k}$, $1 \leq k \leq N$, where $\varepsilon \geq 0$, if for any $i, 1 \leq i \leq N$, we have

$$
J_{i}\left(u_{i}, u_{-i}\right) \leq J_{i}\left(u_{i}^{\prime}, u_{-i}\right)+\varepsilon
$$

when any alternative $u_{i}^{\prime} \in \mathcal{U}_{i}$ is applied by the $i$ th player. 
Theorem 4: Assume (A1)-(A4), $E z_{i}(0)=0$ for all $i$, and $\|\bar{\Gamma}\|<1$, where $\bar{\Gamma}$ is defined by (24). Then given any $\varepsilon>0$, there exists $N_{\varepsilon}$ such that for all $N \geq N_{\varepsilon}$, the set of strategies

$$
\hat{u}_{i}=-R^{-1} B_{i}^{T}\left(\Pi_{\theta_{i}} z_{i}+s_{\theta_{i}, p_{i}}\right), \quad 1 \leq i \leq N
$$

is an $\varepsilon$-Nash equilibrium, where $s_{\theta_{i}, p_{i}}$ is given by (18)-(21) by taking $\theta=\theta_{i}$ and $\alpha=p_{i}$ in $s_{\theta, \alpha}$.

Proof: An outline of the proof is as follows. Given the strategies of all other players, the coupling term in the cost of agent $i$ is stochastic but may be tightly approximated by a deterministic function. Then we further estimate the performance gain of agent $i$ if it switches to another strategy, and the estimate may be facilitated by a standard optimal tracking problem. Now, let $\bar{z}_{\theta, \alpha}$ be given by (18)-(21). Denote

$$
\begin{aligned}
& R_{p_{i}}^{(N)}(t)=\gamma\left[\sum_{j=1}^{N} \omega_{p_{i}, p_{j}}^{(N)} \bar{z}_{\theta_{j}, p_{j}}(t)+\eta\right], \\
& \Delta_{i}^{(N)}(t)=\gamma\left[\sum_{j=1}^{N} \omega_{p_{i}, p_{j}}^{(N)} \bar{z}_{\theta_{j}, p_{j}}(t)-\sum_{j=1}^{N} \omega_{p_{i} p_{j}}^{(N)} z_{j}(t)\right] .
\end{aligned}
$$

We first write the individual cost (13) in the form

$$
\begin{aligned}
J_{i}\left(u_{i}, u_{-i}\right)=E \int_{0}^{\infty} e^{-\rho t} & \left\{\left[\left(z_{i}-R_{p_{i}}^{(N)}\right)+\Delta_{i}^{(N)}\right]^{T} Q\right. \\
\times & {\left.\left[\left(z_{i}-R_{p_{i}}^{(N)}\right)+\Delta_{i}^{(N)}\right]+u_{i}^{T} R u_{i}\right\}(t) d t . }
\end{aligned}
$$

Suppose that all the $N$ agents apply the controls $\hat{u}_{i}, 1 \leq i \leq N$. Denote $\hat{u}_{-i}=\left(\hat{u}_{1}, \ldots, \hat{u}_{i-1}, \hat{u}_{i+1}, \ldots, \hat{u}_{N}\right)$. By the compactness of $\Theta$ in (A3), we may find a constant $\widehat{C}<\infty$ such that

$$
\sup _{N} \sup _{1 \leq i \leq N} E \int_{0}^{\infty} e^{-\rho t}\left(\left|\hat{z}_{i}\right|^{2}+\left|\hat{u}_{i}\right|^{2}\right)(t) d t \leq \widehat{C}
$$

and $J_{i}\left(\hat{u}_{i}, \hat{u}_{-i}\right) \leq \widehat{C}$, where $\hat{z}_{i}$ denotes the state process associated with $\hat{u}_{i}$.

In the analysis below, we consider an alternative strategy $u_{i}$ for agent $i$ while all other agents' strategies are given by $\hat{u}_{-i}$. We may assume that $u_{i}$ satisfies

$$
\begin{aligned}
& E \int_{0}^{\infty} e^{-\rho t}\left[z_{i}-\Phi_{i}\left(z^{(N)}\right)\right]^{T} Q\left[z_{i}-\Phi_{i}\left(z^{(N)}\right)\right] d t \leq \widehat{C} \\
& E \int_{0}^{\infty} e^{-\rho t} u_{i}^{T} R u_{i} d t \leq \widehat{C} .
\end{aligned}
$$

This restriction causes no loss of generality since, otherwise, $u_{i}$ will generate a cost higher than $J_{i}\left(\hat{u}_{i}, \hat{u}_{-i}\right)$. Based on (26), (27) and the observability of $\left[Q^{1 / 2}, A_{i}\right]$, where the associated pair $\left[A_{i}, B_{i}\right]$ is from the compact set $\Theta$, we may further show that $E \int_{0}^{\infty} e^{-\rho t}\left|z_{i}\right|^{2} d t \leq \widehat{C}_{1}$ for some $\widehat{C}_{1}<\infty$ independent of $N$.

By using (A4), for each fixed $t$ we can use the method in [14] to further show

$$
\lim _{N \rightarrow \infty} \sup _{p_{i}}\left|R_{p_{i}}^{(N)}(t)-R_{p_{i}}(t)\right|=0 .
$$

Subsequently, since $\sup _{N} \sup _{p_{i}, t}\left[\left|R_{p_{i}}^{(N)}(t)\right|+\left|R_{p_{i}}(t)\right|\right]<\infty$, by (A3) and boundedness of $R_{p_{i}}(t)$, we may combine (28) with the bounded convergence theorem to show

$$
\lim _{N \rightarrow \infty} E \int_{0}^{\infty} e^{-\rho t} \sup _{p_{i}}\left|R_{p_{i}}^{(N)}(t)-R_{p_{i}}(t)\right|^{2} d t=0 .
$$

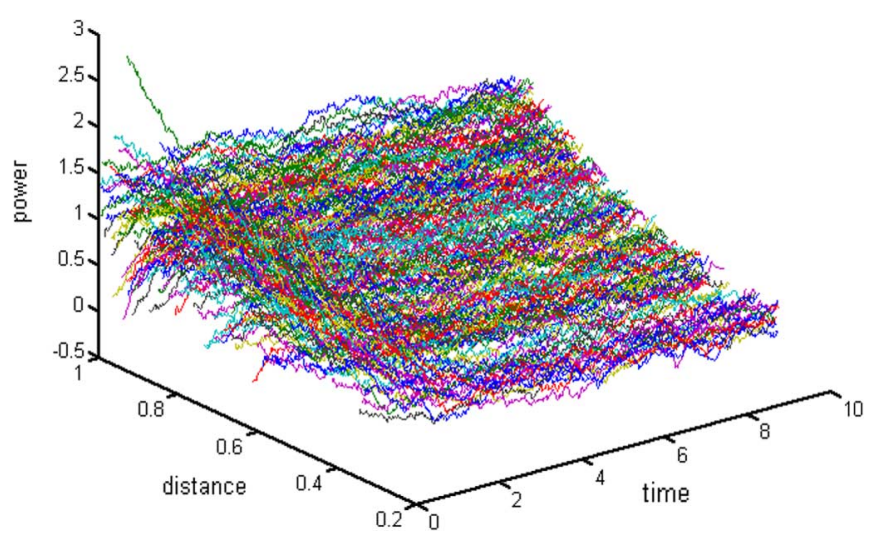

Fig. 1. Trajectories of 200 players.

Then we use (A1)-iii) to establish the convergence relation

$$
\lim _{N \rightarrow \infty} \sup _{u_{i}, i} \int_{0}^{\infty} e^{-p t} E\left|\Delta_{i}^{(N)}(t)\right|^{2}=0
$$

where $u_{i}$ is subject to the constraint (26), (27) and all other agents' strategies are given by $\hat{u}_{-i}$.

Finally, by use of (29), (30) and the approximation argument in [14] we may show that

$$
J_{i}\left(u_{i}, \hat{u}_{-i}\right) \geq J_{i}\left(\hat{u}_{i}, \hat{u}_{-i}\right)-\epsilon_{N}
$$

where $u_{i}$ satisfies (27) and $0 \leq \epsilon_{N} \rightarrow 0$, as $N \rightarrow \infty$. By the choice of $\widehat{C}$, we see that (31) is automatically true when $u_{i}$ does not satisfy (26), (27). This completes the proof.

\section{NUMERICAL EXAMPLE}

We give a numerical example to illustrate decentralized control using locality dependent NCE feedback. We consider a population of $N=$ 200 agents with dynamics given by (9). These agents are distributed uniformly in an annulus of outer diameter $D=2$ and inner diameter $d=2 \sqrt{0.1}$. To reduce the computational load, we adopt a variant of (12) by taking a coupling term of the form $\gamma\left(\sum_{j=1}^{N} \omega_{i j}^{(N)} x_{j}+\eta\right)$. Here we use a normalized but slightly nonlinearly distorted weight allocation $\omega_{i j}^{(N)}$ as specified below. Set $g\left(r_{i}\right)=K r_{i}^{-3}$, where $K>0$ is a coefficient, and let $\omega_{i j}^{0}=g\left(r_{j}\right) / g\left(r_{i}\right)=\left(r_{i} / r_{j}\right)^{3}$. Next, we construct the distortion term $\omega_{i j}^{*}=e^{-\lambda D_{i j}}$, where $D_{i j}$ is the distance between agent $i$ and agent $j$. Finally, we set $w_{i j}^{(N)}=c_{i}\left(\omega_{i j}^{0}+\omega_{i j}^{*}\right)$, where $c_{i}$ is a normalizing factor such that $\sum_{j=1}^{N} \omega_{i j}^{(N)}=1$. We take the parameters $\rho=0.5, \gamma=0.5, r=1, \eta=2, \sigma=0.15$, and $\lambda=5$. The initial means of all $x_{i}$ 's are equal to 1 .

The associated NCE equation system is numerically solved with a time step size of 0.02. The decentralized control law is applied, and Fig. 1 shows the individual trajectories on the time interval $[0,10]$. It is seen that for agents closer to the outer boundary of the annulus, the controlled powers are higher in average.

\section{CONCLUSION}

In this technical note we have generalized our previous Nash Certainty Equivalence methodology with uniform coupling to models with locality interactions where the weight allocation in the cost coupling affects the spatial spreading ability of interactions. It has been shown that for reasonably slow decay rates on the interaction strength, a consistency relationship between individual strategies and local deterministic mass effects can still be specified, and this procedure leads to decentralized $\varepsilon$-Nash strategies for the individual players. 


\section{APPENDIX}

Proof of Lemma 1: Given $f \in C_{b}\left[\mathcal{C} \times \mathbb{R}^{+}\right]$, we have

$$
\begin{aligned}
{\left[\Gamma_{\theta} f(\alpha, \cdot)\right](t)=\int_{0}^{t} e^{A_{1}(t-s)} B R^{-1} B^{T} } & e^{-A_{2}^{T} s} \\
& \times \int_{s}^{\infty} e^{A_{2}^{T} \tau} \gamma[f(\alpha, \tau)+\eta] d \tau d s .
\end{aligned}
$$

By the boundedness of $f(\alpha, t)$ and (A3), there exists $C<\infty$ such that $\sup _{\alpha, t}\left|\left[\Gamma_{\theta} f(\alpha, \cdot)\right](t)\right| \leq C$, where $C$ may be chosen to be dependent on $|f|=\sup _{\alpha, t}|f(\alpha, t)|$, but independent of $\theta$. Subsequently

$$
\begin{aligned}
\sup _{\alpha, t}|(\Gamma f)(\alpha, t)| & \leq \sup _{\alpha, t} \int_{\theta \in \Theta} \int_{\alpha^{\prime} \in \mathcal{C}}\left|\left[\Gamma_{\theta} f\left(\alpha^{\prime}, \cdot\right)\right](t)\right| d F(\theta) d F_{\alpha}\left(\alpha^{\prime}\right) \\
& \leq C
\end{aligned}
$$

and hence $\Gamma f$ is bounded. Now for a given $\theta$, we prove the continuity of $\Gamma f$. We note the relation

$$
\begin{aligned}
{\left[\Gamma_{\theta} f(\alpha, \cdot)\right](t)=} & \int_{0}^{t} e^{A_{1}(t-s)} B R^{-1} B^{T} e^{-A_{2}^{T} s} \\
& \times \int_{s}^{\infty} e^{A_{2}^{T} \tau} \gamma(f(\alpha, \tau)+\eta) d \tau d s \\
= & \gamma e^{A_{1} t} \int_{0}^{t} e^{-A_{1} s} B R^{-1} B^{T} \\
& \times \int_{s}^{\infty} e^{A_{2}^{T}(\tau-s)} f(\alpha, \tau) d \tau d s+\Phi(t)
\end{aligned}
$$

where $\Phi(t)$ is a continuous function of $t$. Define

$$
\begin{aligned}
G_{0}(\theta, \alpha, t) & =\int_{0}^{t} e^{-A_{1} s} B R^{-1} B^{T} \int_{s}^{\infty} e^{A_{2}^{T}(\tau-s)} f(\alpha, \tau) d \tau d s, \\
G(\alpha, t) & =\int_{\theta \in \Theta} \int_{\alpha^{\prime} \in \mathcal{C}} G_{0}\left(\theta, \alpha^{\prime}, t\right) d F_{\alpha}\left(\alpha^{\prime}\right) d F(\theta) .
\end{aligned}
$$

Now to prove the continuity of $\Gamma f$, it suffices to show the continuity of $G(\alpha, t)$ with respect to $(\alpha, t)$. Letting $(\alpha, t)$ be fixed, we pick $\left(\alpha_{1}, t_{1}\right)$ in a neighborhood of $(\alpha, t)$. Then

$$
\begin{aligned}
\left|G\left(\alpha_{1}, t_{1}\right)-G(\alpha, t)\right| \leq\left|G\left(\alpha_{1}, t_{1}\right)-G\left(\alpha_{1}, t\right)\right| & \\
& +\left|G\left(\alpha_{1}, t\right)-G(\alpha, t)\right| .
\end{aligned}
$$

We have

$$
\begin{aligned}
& \left|G\left(\alpha_{1}, t_{1}\right)-G\left(\alpha_{1}, t\right)\right| \\
& \leq \int_{\theta} \int_{\alpha^{\prime}}\left|\int_{t}^{t_{1}} e^{-A_{1} s} B R^{-1} B^{T} \int_{s}^{\infty} e^{A_{2}(\tau-s)} f\left(\alpha^{\prime}, \tau\right) d \tau d s\right| \\
& \quad \times d F_{\alpha_{1}}\left(\alpha^{\prime}\right) d F(\theta) \\
& \leq \int_{\theta} \int_{\alpha^{\prime}} C\left|t_{1}-t\right| d F_{\alpha_{1}}\left(\alpha^{\prime}\right) d F(\theta)=C\left|t_{1}-t\right|
\end{aligned}
$$

for some $C<\infty$ depending only on $|f|$ and $\Theta$.

$$
\text { Denote } G_{1}(\alpha, t)=\int_{\theta} G_{0}(\theta, \alpha, t) d F(\theta) \text {. We have }
$$

$$
\begin{aligned}
\left|G\left(\alpha_{1}, t\right)-G(\alpha, t)\right| \leq \mid \int_{\alpha^{\prime}} G_{1}\left(\alpha^{\prime}, t\right) d F_{\alpha_{1}}\left(\alpha^{\prime}\right) & \\
& -\int_{\alpha^{\prime}} G_{1}\left(\alpha^{\prime}, t\right) d F_{\alpha}\left(\alpha^{\prime}\right) \mid .
\end{aligned}
$$

For each fixed $t, \sup _{\alpha^{\prime}}\left|G_{1}\left(\alpha^{\prime}, t\right)\right|<\infty$ and by elementary estimates we can show that $G_{1}\left(\alpha^{\prime}, t\right)$ is a continuous function of $\alpha^{\prime}$. Hence from the weak convergence of $F_{\alpha_{1}}$ to $F_{\alpha}$ when $\alpha_{1} \rightarrow \alpha$, it follows that:

$$
\lim _{\alpha_{1} \rightarrow \alpha}\left|G\left(\alpha_{1}, t\right)-G(\alpha, t)\right|=0 .
$$

Finally, the continuity of $G(\alpha, t)$ follows from (A1) and (A2). The lemma follows.

\section{ACKNOWLEDGMENT}

The authors wish to thank A. Lazarte for the observation following equations (18)-(21), and for providing the simulation example.

\section{REFERENCES}

[1] G. A. Akerlof, "Social distance and social decisions," Econometrica, vol. 65, no. 5, pp. 1005-1027, Sep. 1997.

[2] T. Başar and G. J. Olsder, Dynamic Noncooperative Game Theory, 2nd ed. London, U.K.: Academic Press, 1995.

[3] R. Balescu, Statistical Dynamics: Matter Out of Equilibrium. London, U.K.: Imperial College Press, 1997.

[4] C. T. Bauch and D. J. D. Earn, "Vaccination and the theory of games," Proc. Natl. Acad. Sci., vol. 101, pp. 13391-13394, Sep. 2004.

[5] P. Billingsley, Convergence of Probability Measures, 2nd ed. New York: Wiley, 1999.

[6] A. Bisin, U. Horst, and O. Özgür, "Rational expectations equilibria of economies with local interactions," J. Econ. Theory, vol. 127, pp. 74-116, 2006

[7] L. E. Blume, "The statistical mechanics of strategic interaction," Games Econ. Behavior, vol. 5, pp. 387-424, 1993.

[8] R. Breban, R. Vardavas, and S. Blower, "Mean-field analysis of an inductive reasoning game: Application to influenza vaccination," Phys. Rev. E, vol. 76, no. 3, Sep. 2007.

[9] D. A. Dawson and J. Gärtner, "Large deviations from the MckeanVlasov limit for weakly interacting diffusions," Stochastics, vol. 20, pp. 247-308, 1987.

[10] G. M. Erickson, "Differential game models of advertising competition," Eur. J. Oper. Res., vol. 83, pp. 431-438, 1995.

[11] E. J. Green, "Continuum and finite-player noncooperative models of competition," Econometrica, vol. 52, no. 4, pp. 975-993, 1984.

[12] U. Horsta and J. A. Scheinkman, "Equilibria in systems of social interactions," J. Econ. Theory, vol. 130, no. 1, pp. 44-77, 2006.

[13] M. Huang, P. E. Caines, and R. P. Malhamé, "Individual and mass behaviour in large population stochastic wireless power control problems: Centralized and Nash equilibrium solutions," in Proc. 42nd IEEE Conf. Decision Control, Maui, HI, Dec. 2003, pp. 98-103.

[14] M. Huang, P. E. Caines, and R. P. Malhamé, "Large-population costcoupled LQG problems with nonuniform agents: Individual-mass behavior and decentralized $\varepsilon$-Nash equilibria," IEEE Trans. Autom. Control, vol. 52, no. 9, pp. 1560-1571, Sep. 2007.

[15] M. Huang, P. E. Caines, and R. P. Malhamé, "The Nash certainty equivalence principle and Mckean-Vlasov systems: An invariance principle and entry adaptation," in Proc. 46th IEEE Conf. Decision Control, New Orleans, LA, Dec. 2007, pp. 121-126.

[16] M. Huang, R. P. Malhamé, and P. E. Caines, "Nash equilibria for large-population linear stochastic systems of weakly coupled agents," in Analysis, Control and Optimization of Complex Dynamic Systems, E. K. Boukas and R. P. Malhamé, Eds. New York: Springer, 2005, pp. 215-252.

[17] M. Huang, R. P. Malhamé, and P. E. Caines, "Large population stochastic dynamic games: Closed-loop Mckean-Vlasov systems and the Nash certainty equivalence principle," Commun. Inform. Syst., vol. 6, no. 3, pp. 221-252, 2006. 
[18] M. Huang, R. P. Malhamé, and P. E. Caines, "Nash certainty equivalence in large population stochastic dynamic games: Connections with the physics of interacting particle systems," in Proc. 45th IEEE CDC Conf., San Diego, CA, Dec. 2006, pp. 4921-4926.

[19] M. A. Khan and Y. Sun, "Non-cooperative games with many players," in Handbook of Game Theory With Economic Applications, R. J. Aumann and S. Hart, Eds. North-Holland, The Netherlands: Elsevier Science, 2002, vol. 3, pp. 1761-1808.

[20] V. E. Lambson, "Self-enforcing collusion in large dynamic markets," J. Econ. Theory, vol. 34, pp. 282-291, 1984.

[21] J.-M. Lasry and P.-L. Lions, "Jeux à champ moyen. I-le cas stationnaire," C. R. Acad. Sci. Paris, Ser. I, vol. 343, pp. 619-625, 2006.

[22] J.-M. Lasry and P.-L. Lions, "Mean field games," Jpn. J. Math., vol. 2, no. 1, pp. 229-260, 2007

[23] T. Li and J.-F. Zhang, "Asymptotically optimal decentralized control for large population stochastic multiagent systems," IEEE Trans. Autom. Control, vol. 53, no. 7, pp. 1643-1660, Jul. 2008.

[24] M. A. Nowak and K. Sigmund, "Games on grids," in The Geometry of Ecological Interactions: Simplifying Spatial Complexity, U. Dieckmann, R. Law, and J. A. J. Metz, Eds. Cambridge, U.K.: Cambridge Univ. Press, 2000, pp. 135-150.

[25] T. C. Reluga and S. Viscido, "Simulated evolution of selfish herd behavior," J. Theor. Biol., vol. 234, pp. 213-225, 2005.

[26] T. C. Schelling, "Dynamic models of segregation," J. Math. Soc., vol. 1, pp. 143-186, 1971

[27] A.-S. Sznitman, "Topics in propagation of chaos," in Ecole d'Eté de Probabilitiés de Saint-Flour XIX-1989, Lect. Notes Math. 1464. Berlin, Germany: Springer-Verlag, 1991, pp. 165-252.

[28] C. T. Thompson, Classical Equilibrium Statistical Mechanics. Oxford, U.K.: Clarendon Press, 1988.

[29] G. Y. Weintraub, C. L. Benkard, and B. V. Roy, "Oblivious equilibrium: A mean field approximation for large-scale dynamic games," in Advances in Neural Information Processing Systems. Cambridge, MA: MIT Press, 2005.

[30] G. Y. Weintraub, C. L. Benkard, and B. V. Roy, "Markov perfect industry dynamics with many firms," Econometrica, vol. 76, no. 6, pp. 1375-1411, Nov. 2008.

[31] H. Tembine, J.-V. Le Boudec, R. El-Azouzi, and E. Altman, "Mean field asymptotics of Markov decision evolutionary games and teams," in Proc. Int. Conf. Game Theory Networks, May 2009, pp. 140-150.

\section{Three-Dimensional Motion Coordination in a Spatiotemporal Flowfield}

Sonia Hernandez and Derek A. Paley, Member, IEEE

Abstract-Decentralized algorithms to stabilize 3-D formations of unmanned vehicles in a flowfield that varies in space and time have applications in environmental monitoring in the atmosphere and ocean. In this note, we provide a Lyapunov-based control design to steer a system of selfpropelled particles traveling in three dimensions at a constant speed relative to a spatiotemporal flowfield. We assume that the flow is known locally to each particle and that it does not exceed the particle speed. Multiple particles can be steered to form 3-D parallel or helical formations in a flowfield. Also presented are motion coordination results for a special case of the 3-D model in which the particles travel in a circular formation on the surface of a rotating sphere.

Index Terms-Cooperative systems, multiagent systems, nonlinear control systems.

\section{INTRODUCTION}

Decentralized algorithms to stabilize collective motion in a 3-D flowfield that varies in space and time can be applied in many real-world scenarios [1], [2]. Previous work on collective motion in a flowfield has focused on a planar model of self-propelled particles [2]-[4], which is sufficient for studying motion coordination in a two-dimensional operational domain. Most prior work on non-planar collective motion has focused on flow-free models [5]-[7]. We provide a Lyapunov-based control design to steer a system of self-propelled particles traveling in three dimensions at a constant speed relative to a spatiotemporal flowfield. We assume that the flow is known locally to each particle and that it does not exceed the particle speed. Our model of 3-D motion coordination is motivated by unmanned vehicles that operate in a 3-D domain — such as underwater gliders [8] and long-endurance aircraft [9]. Motivated by constant-altitude/-depth surveys over spatial scales for which the curvature and/or rotation of Earth are relevant, we also study a special case of the 3-D model in which particles are constrained to the surface of a rotating sphere.

Our analysis extends [6] and [10], which describe decentralized strategies to steer a 3-D system of self-propelled particles in a flow-free environment. It also extends the 3-D analysis in [11], which includes a spatially variable, time-invariant flowfield. To model a spatiotemporal flowfield in three dimensions, we adapt the development of a planar framework for collective motion in a time-varying flow [2]. The spherical analysis extends [7], which introduced a flow-free spherical model. Additional prior work appears in [12] and an extended presentation of these results is available [13]. The study of motion coordination in an unknown flowfield or with turn-rate limits is ongoing [14] and outside the scope of this note, as is the study of flowfields that exceed the particle speed.

The contribution of this note is the Lyapunov-based design of decentralized control laws to stabilize moving formations of a 3-D, connected

Manuscript received September 01, 2009; revised September 02, 2009, April 14, 2010; accepted August 16, 2010. Date of publication September 02, 2010; date of current version December 02, 2010. Recommended by Associate Editor M. Egerstedt.

S. Hernandez is with the Department of Aerospace Engineering, The University of Texas at Austin, Austin, TX 78712 USA (e-mail: sonia.hernandez@mail. utexas.edu).

D. A. Paley is with the Department of Aerospace Engineering, University of Maryland, College Park, MD 20742 USA (e-mail: dpaley@umd.edu).

Digital Object Identifier 10.1109/TAC.2010.2070710 